\title{
Links Between Creative Performance and Post-Formal Thought
}

\author{
Asli Nazife Kaya \\ Izmir Institute of Technology, Turkey \\ E-mail address: n.aslikaya@gmail.com
}

\author{
Seniz Cikis \\ Izmir Institute of Technology, Turkey \\ E-mail address: senizcikis@ @iyte.edu.tr
}

\section{ARTICLE INFO}

\section{Keywords:}

Creative performance

Design

Post-formal thought

\section{Article history:}

Received 12 February 2017

Received in revised form 21 April 2017

Accepted 25 April 2017

ISSN: 2354-0036

DOI: $10.1515 /$ ctra-2017-0006

\section{A B STRACT}

The aim of this study is to reveal relations between creativity in design and the cognitive developmental stages of designers within a Post-Piagetian approach. In this study design processes were deeply inspected with the aim of obtaining more comprehensive information about the effects of individual's cognitive status on the design processes, problemsolving and quality of idea generation. In this field, studies done so far have been conducted on test results as indicators of creativity. Withinthe present study, however, protocol analyses were also evaluated, in addition to test results, and thanks to this addition, it was possible to determine the qualitative and quantitative effects of cognitive developmental stages on creativity. A total of twenty-five students voluntarily participated in the research. To collect data, two different methods were employed. The Social Paradigm Belief Inventory (SPBI), was employed to determine students' cognitive stages and scores, and the Think-aloud protocol was used to elicit verbal reports of the thought sequences of the students. In order to analyse the verbal reports reflecting designers' cognitive activities, the method of Linkography was conducted and the general statistics of segments were measured. To assess the relations between creativity in design and the cognitive developmental stages of the designers, SPBI scores and the general statistical values obtained from the design sessions were correlated. Findings showed that there is a strong and significant correlation between the cognitive development levels and creativity, in favour of dialectical thinking.

\section{INTRODUCTION}

Creativity has always been a major topic of design research, owing to its crucial role in the design and problem-solving process (Mumford et al., 1991; Runco, 1994; Torrance, 1984; Torrance, 1988; Wallas, 1926). Therefore, research on creativity and its sub-topics cover an extremely broad array of subjects. One of these research sub-topics focuses 
on cognition and its influences on creative behaviour and creative potentials. Creativity is defined as combining, synthesising and/or bridging already existing ideas, information and /or knowledge in order to generate novel, useful and appropriate solutions, and the creative process is defined as creative thinking in terms of an individual internal cognitive process. Koestler (1960) describes the creative process as connecting previously unrelated matrices of thought and generating new ideas. The individual consciously or unconsciously starts exploring knowledge units to discover how they could be linked to each other and how to map them. This mapping allows for new connections in order to solve a problem. In short, according to this approach, a transformation occurs through linking knowledge units, that are associated with each other, or forming new connections between independent knowledge units (Boden, 1994; Gabora, 2002; Santanen et al, 2002, Ward et al, 1997) and this transformation gives rise to novel applicable solutions (Gabora, 2002; Santanen et al, 2002). This process demands an ability to perceive actual or potential relations between knowledge units and to link them in order to generate ideas.

Akin and Akin (1996), suggested establishing the importance of sudden mental insight in breaking through fixation and establishing a new frame of reference to generate a novel solution to a problem. Cross (1997), analysed the design protocols of a design team to identify the role of the creative event that occurs during the problem-solving processes, and how this creative event bridges problem space and solution space. Van der Lugt (2003), accepted idea quality as an indicator of creativity and claimed that "wellintegrated idea generation is an indicator for quality of process" (p.1). Similarly, Goldschmidt and Tatsa (2005) and Tatsa (2005) have shown that ideas heading to novel solutions tend to have plentiful interconnections.

These concepts originating from different researchers can be seen as pieces of a puzzle; sudden mental insight to break through, bridging problem space and solution space, good integration of sub-ideas and making plentiful interconnections between design moves; the key to this puzzle might lie in the cognitive development stages where each of these concepts is a feature of the stages. As Runco (2004) states, creative development is closely connected to cognitive development. Thus, the main hypothesis for this research is the assumption that finding a creative solution to a design problem requires advanced, rather than formal thinking skills, which are linked with post-formal thought stages, and creative performance has strong connections with the thinking types of the post-formal process; i.e. relativistic and dialectical. 
Post-formal thought is a cognitive stage beyond Piaget's formal operations stage ${ }^{1}$. As the individual's cognition develops, his or her thinking and understanding of the world becomes more abstract and complex, and the ability for holistic thinking develops in the late formal thought stages. The formal thought stage, also known as the formal operational stage or hypothetico-deductive stage, is the fourth phase in Piaget's theory and starts between ages 11 to 15 (Piaget, 1958). For most adolescents, cognitive development stops at this stage, as they think holistically, logically and abstractly and do not need concrete examples anymore while operating. Here, abstract thinking refers to abstraction as in mathematics, where a concrete reality can be unequivocally represented by a symbol, thus problems can only have one true solution. At the formal thought stage, the individual can only find a solution within a closed system and deal with well-defined problems including controllable, specific variables -which make it ill-suited for design problems characterised by complex variables, incomplete data and uncertain outcomes. Thus, formal thinking is better applied to problems involving, scientific, logical and mathematical thinking than illdefined design problems. While success in natural sciences is closely correlated with the formal thinking ability (Mwamwenda, 1993; Valanides, 1998), design demands creativity and holistic thinking that characterise the post-formal thought stage (Wu \& Chiou, 2008).

Formal thinking is unable to solve design problems effectively, as these are openended and difficult to solve in a systematic, logical and methodical way, and thus formal thinking falls short in the context of developmental-appropriateness. Two pioneers in the research on post-formal thinking, Commons and Richards (2003) explain: "The term "postformal" has come to refer to various stage characterizations of behaviour that are more complex than those behaviours found in Piaget's last stage - formal operations and generally seen only in adults." (p.199). According to Commons et al. (2008), postformal thought places a greater emphasis on both problem solving and problem finding. According to Schön (1983), the "problem of design problem" (p.129) is finding out what the problem actually is. With Cross's (2008) words; the "mysterious, creative part of designing" (p.40) is the ability to find problems, defining possible solutions and using a particular way of thinking. Indeed, finding the design problem is not enough to solve it, the designer also needs to reframe it in order to solve it. The post-formal thought ability allows the individual to reconceptualise problems from different perspectives. Thus, the individual at the post-formal thinking stage can solve open-ended, complex problems in addition to creating novel relations between knowledge chunks (Commons et al., 2008), and can apply relativistic and/or dialectic thinking to problem-solving.

\footnotetext{
${ }^{1}$ Although post-formal thought is widely accepted as the fifth stage of cognitive development, there have been criticisms of this conceptualisation. For further information please see Marchand (2002) and McBride (1998).
} 
In relativistic thinking, individuals are aware that the nature of knowledge is not dualistic (Kramer, 1983), the nature of truth is relative (Perry, 1981), and problems can be viewed from many perspectives (Wu P-L. \& W-B. Chiou, 2008). According to Basseches (1984), the relativistic thinker assumes that there are multiple subjective realities, thus there cannot be a universal truth. Labouvie-Vief (1980) points out that the autonomous (relativistic) thinker can take subjective criteria into consideration while reasoning. As an example (Kramer, 1992), relativistic thinkers accept "there are no absolute moral principles. This is because morality is personal, and people have different ideas about what morality is." (p.1). Thus, relativistic thinkers can create new ideas by linking knowledge units within a dependently changing thought process. This thinking ability can generate more interconnections between ideas than absolute thinking. Schön's rigour vs relevance concept can be applied to our understanding of the relativistic thought stage. According to Schön, the dilemma of rigor or relevance arises from the insistence on solving real-life problems in a closed-system by applying technical knowledge obtained from theory-based research and techniques to ill-defined problems (Schön, 1983). But, as is well-known, reallife problems cannot be easily solved by exclusively applying a 'rational' or 'scientific' approach. Indeed, this kind of insistence is closely related to formal thought. In contrast with the rigorousness of formal thought, relativistic thinking values relevance; it operates in open-systems and allows individuals to think in more complex ways. Thus, according to Wu and Chiou (2008), relativistic thinking is a source of greater diversity and novelty.

However, relativistic thinking doesn't allow the individual to link knowledge across multiple perspectives at once. To do this, the individual should have the ability to think dialectically. Dialectical thinking is considered the most advanced form of post formal thought (Laske, 2009). According to Basseches (1984), a dialectical thinker is able to apply relativistic and formal logic in a new way by focusing on the process itself. The individuals at the dialectical thought stage are aware that the so-called truth is valid within a specific ideology, but might not be valid in a different reality, thus they apperceive there are multiple 'right' answers (Labouvie-Vief, 1982). As an example (Kramer, 1992), dialectical thinkers accept "there are non-absolute moral principles. This is because we each form a set of consistent rules to guide our lives, which makes the most sense in terms of our overall life goals" (p.1). By realising there are multiple realities and truths, dialectical thinkers become aware that all phenomena and their opposites are connected to each other (Kramer, 1989). In dialectic thinking, relativism integrates into the ability to conceptualise contradictions as interrelated and part of a whole. "Reflection for a dialectical thinker is the process of taking one idea, then reflecting upon its opposite or reflecting up- 
on what is left out. The dialectical thinker thus becomes aware of the process of inquiry" (McBride, 1998, p.22). As for dialectical thinking, as identified in Schön's dialectic between design and designer, individuals are aware of their thoughts and the fact that these are in a constant process of evolution (Basseches, 1989). Thus, dialectical thinkers are aware of their ability to break open commons and/or traditions and act more creatively. Dialectical thinkers tend to discuss a problem from different and even opposite perspectives and find solutions by integrating these into a thesis and anti-thesis. Therefore, dialectical thinking ability may lead to many more interconnections between ideas than formal and relativistic thinking.

However, as of yet, little research has focused on the links between creativity and post-formal thinking stages. In 2008, Wu and Chiou investigated the relationship between post-formal thought levels and creative performance among late adolescents. They employed the Divergent Thinking Test to measure creative performance and the Social Paradigm Belief Inventory to measure participants' thinking levels. The results of their study showed that there is a strong, positive relationship between dialectical and relativistic thinking, and creativity, whereas formal thinking was negatively correlated. Yang et al. (2010), similarly, examined the connection between the parameters of creativity and dialectical thinking, applying the same method as Wu and Chiou (2008). They also concluded that cognitive development and creativity are closely related and dialectical thinking may be the catalyser of creative performance. Blouin and McKelvie (2012) researched the connection of post-formal thinking with metaphor and irony as predictors of creativity. By employing the Social Paradigm Belief Inventory, Irony Test and the Divergent Thinking Test, they demonstrated that post-formal thinking scores are good predictors of creativity and are also correlated with the identification and subjective appreciation of metaphor and irony, whereas formal thinking was correlated negatively.

As presented above, all previous research investigated the relationships between creativity and post-formal thinking by comparing test results. In this research, to acquire a deeper understanding of the relationships between creativity in design and post-formal thinking, a different approach was adopted; data were obtained by analysing the design sessions' protocols and compared with the results from the Social Paradigm Belief Scale, in order to identify how post-formal thinking levels affect idea generation quality and problem-solving skills in the context of creativity.

\section{METHODOLOGY}

A total of twenty-five students, fifteen males and ten females, aged between 20 and 27, with a mean age of 21.52 years, volunteered for this research. All participants were un- 
dergraduate students of the Izmir Institute of Technology, Department of Architecture, in Turkey. $32 \%$ of them were in their $2^{\text {nd }}$ year, $24 \%$ in their $3^{\text {rd }}$ year and $44 \%$ in their $4^{\text {th }}$ year. First year students were not included in the study, as they had not completed the Basic Design Course. The participants were recruited through posting on announcement boards in the Faculty of Architecture.

Data were collected through two different methods. The Social Paradigm Belief Inventory (SPBI) was used to determine students' cognitive stages and scores, and the Think-Aloud Protocol was used to elicit verbal reports of thought sequences as a data source on the students' thinking process during 30 minute design sessions. The data were analysed with the help of LINCODER software developed by Pourmohamadi and Gero (2011). Pearson's correlation coefficient method was used to assess associations between linkographic entropies and SPBI scores with the aid of SPSS v13 software.

\section{Social Paradigm Belief Inventory (SPBI):}

Only two cognitive stage measurements exist: The Complex Post-formal Thought Questionnaire (CPTQ) developed by Sinnott (1989), which includes 10 items describing particular operations of post-formal thought. However, no research has been done on the reliability and validity of CPTQ. The second measurement procedure is the Social Paradigm Belief Inventory (SPBI), developed by Kramer et al. (1992). The SPBI scale has shown internal consistencies ranging from .60 to $.84(M=.72 ; S D=.11)$. Since only the SPBI has been evaluated for reliability, it was used in this study.

The SPBI is a 27-item, forced-choice inventory in which subjects choose one of three statements representing absolute, relativistic or dialectical thinking processes, with which they agree the most. The Absolute statements are indicators of formal operational thought. The Absolute statements are uncontradictory and based on universally wrong or right judgements. The Relativistic and the Dialectical statements are not based on moral universalism. Relativistic statements were based on judgements were derived from personal experiences, knowledge and points of view (Kramer et al., 1992; Basseches, 1984). Thus, contrary to the absolute thinkers, the relativistic thinkers avoid predictions (Kramer et al., 1992). Dialectical statements were based on judgements derived from the evolution of contradictory thoughts and/or pre-judgements (Basseches, 1984), and "it is believed that perspectives always grow and change" (Vanier \& Searight, 2013, p.50). Examples of each paradigm are given below (Kramer et al., 1992, p.6):

Absolute Statement: Solving problems requires quickly coming up with the best solution. This is because there is a correct way of doing things, and a good decision maker, recognising this, decisively wastes no time putting it into action. 
Relativistic Statement: Solving problems requires realising that there is no right solution. This is because there are many different sides of a problem and depending on what side you look at, a good decision maker needs to recognise that there are different solutions.

Dialectic Statement: Problem-solving is a question of developing new perspectives. This is because a good decision maker is able to see many sides of a problem and encourage a dialogue in which everyone will be heard and contribute to each other's thinking.

To get the final scores, for every absolute statement the participant received one point, for every relativistic statement the participant received two points, and for every dialectical statement, the participant received three points. Scores are then summed for a total score, and to find the average, the final sum is divided by 27 . The average scores indicate the frequency with which a participant applies a thinking style. For example, if a participant scores 1.19 points, the researcher can conclude that the participant tends to think in absolute terms, and therefore she is an absolute thinker. So, average scores vary between 1 to 3 points on the scale. Also, SPBI scores are ipsative, in other words, getting high scores on absolute thinking means getting low scores on relativistic and dialectical thinking (Vanier and Searight, 2013).

\section{Think-aloud protocol and Design Sessions:}

The Think-Aloud Protocol (TAP) is a very direct method for gathering raw data on the problem-solving and reasoning processes frequently used by researchers to understand the cognitive processes of designing (Someren et al., 1994; Gursoy, 2010).

To collect data with TAP, the subject is asked to verbalise whatever crosses her / his mind while performing the given task within a timeframe. Meanwhile, the researcher records the activity through appropriate devices without interrupting the subject's task. However, owing to the intensity of the cognitive processes involved, the subject may start muttering or stop speaking altogether; in such circumstances, the researcher should intervene and encourage the subject to speak or raise his / her voice, using as few words as possible (Someren et al., 1994).

As part of this research, a design brief was given to participants in order to collect verbal data. The task was designing a seating unit, to be placed along the Izmir seafront, within a thirty (30) minute timeframe. Participants were given all necessary details about how to conduct the think-aloud method and about the materials available for use during the design session. Each design session was carried out individually, under the nonintrusive observation of the researcher. The design sessions were recorded with a camrecorder and a tape-recorder. 


\section{Linkography:}

Linkography is a method used to analyse designers' cognitive activities by graphically representing moves and the links between them. In order to draw linkographs, data is obtained through think-aloud protocols and segmented into small units, called design moves, based on the design sessions' phonetic transcriptions. Goldschmidt (2014), the main developer of Linkography, defines a design move as "a step, an act, and an operation that transforms the design situation somewhat relative to the state it was in before that move." (p.42). Segmentation of the data was conducted based on this description by three judges. As an example, a participant's segmentation partially is given below:

Move6 I guess... My concept will be nature, as the seaside connotes nature for me

Move7 That's why it [seating unit] shouldn't have orthogonal forms like I am drawing at the moment

Move8 It should have some organic forms like we encounter in nature

Move9 Indeed, just today we researched whether there are any orthogonal forms, as well as organic forms in nature

Move10 It can be something like a honeycomb. This is the simplest one I can think of now

Move 11 Linear like this (pointing to the drawing)... In this way, it can be selfsupportive without the need for another structure

Move12 Also, it can repeat itself and enlarge

Move13 I mean... when we combine the repetitive modules, we can create an organic shape

Move14 When we inspect a leaf we can see how linear forms can create organic forms, so squares can create organic forms... I can use this...

Move 15 Or... I can use triangles as the base element of my design 


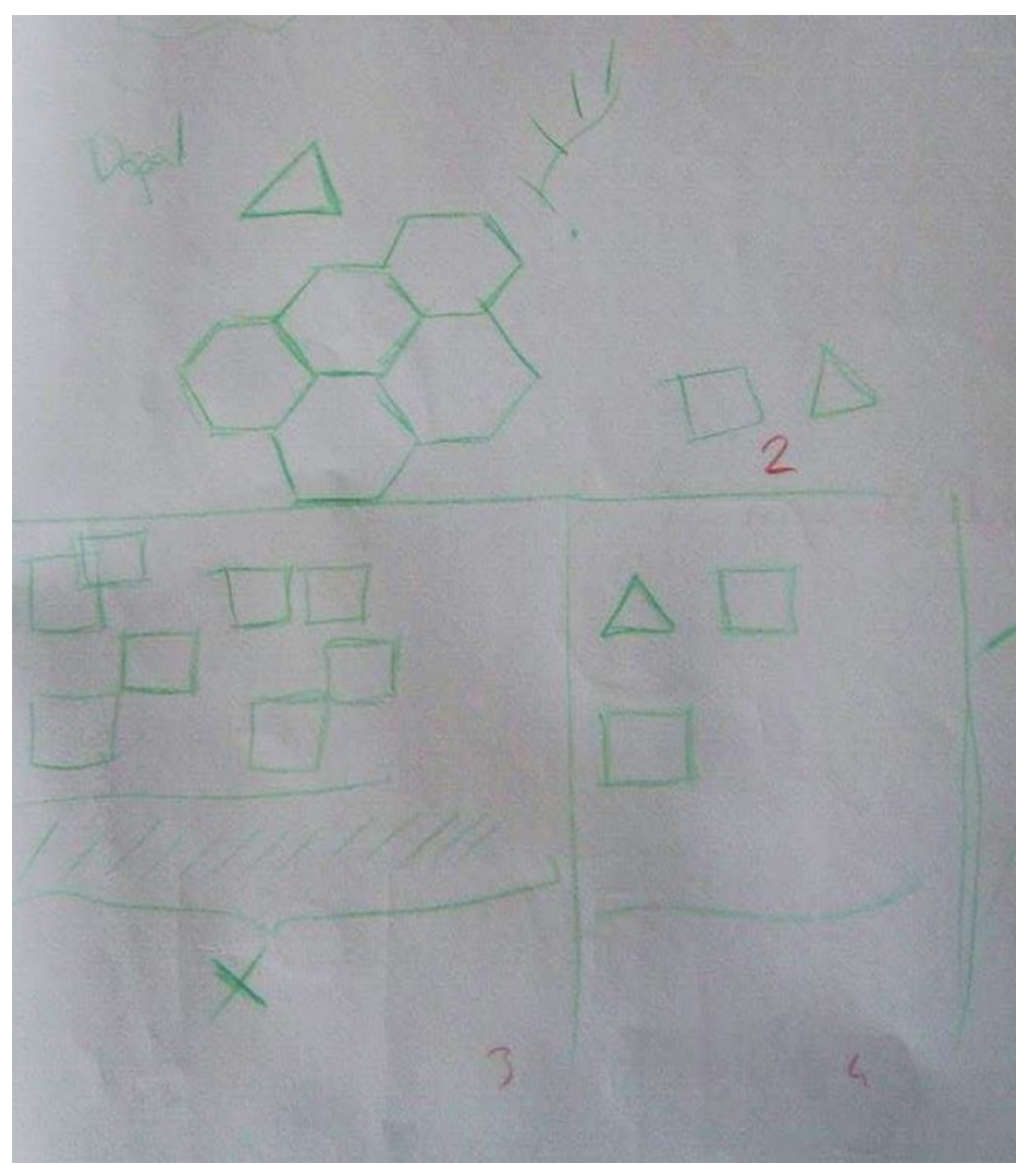

Figure 1. Sketch of the participant, segmentation partially are given above.

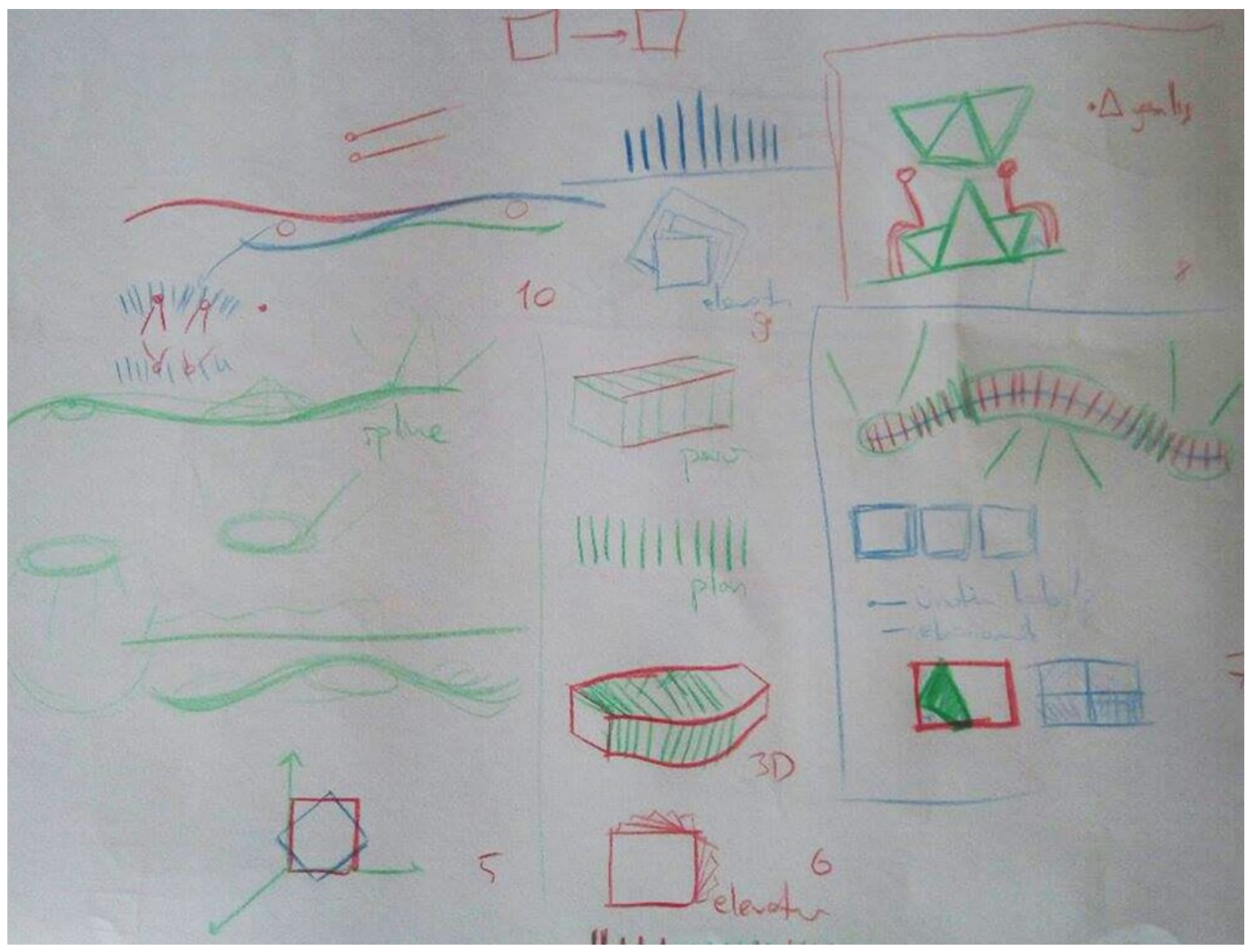

Figure 2. Sketch of the participant, segmentation partially are given above. 
Afterwards, connections between design moves were defined to form links between them. The final linkographs and segments' general statistics, that is the link index and the linkographic entropies, were acquired with the help of the LINCODER software developed by Pourmohamadi and Gero (2011) (Please see Figure 3).

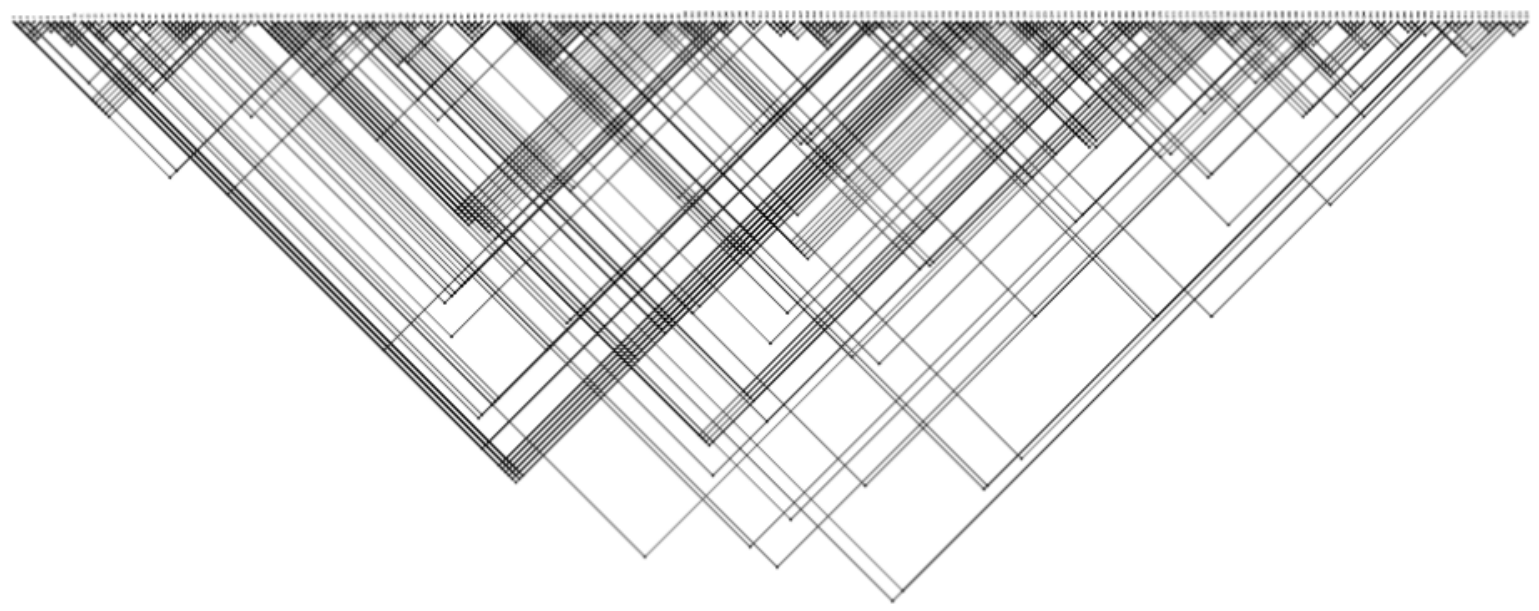

Figure 3. Linkograph of participant 24.

The link index is the ratio between the number of links and the number of moves that generate them in a given design session (Goldschmidt, 2014). Where numbers of design moves are higher, the link index will be higher too. The link index value, prima facie, can be assumed to be a kind of pre-indicator of the amount of linking activity, which is also an indicator of the designer's effort to achieve a synthesis (Goldschmidt, 2014). Thus, in this study, the link index is accepted as a variable of linking productiveness.

According to Goldschmidt (2014), "critical moves" (CM), which are rich in links, are one of the main indicators of creativity. Critical moves (CMs) are the design moves outnumbering the majority of the moves in a design session on forming links. The critical moves have significant roles in determining creativity because these moves are the indicators of quality of idea generation and problem-solving process (Goldschmidt, 2014). Thus, in this study, CMs are accepted as a variable of creativity. Research has been showed that critical moves represent a minimum of $10 \%$ to $12 \%$ of an individual's whole problem-solving thought process. As part of this research, the link number threshold for CMs was set at 6.

\section{RESULTS}

Table 1 shows the results of the SPBI scores and the Linkographic entropies. As seen from the table, there is a positive linear relationship between the SPBI scores and the link index. Also, as the SPBI score increase, the critical moves, which are the main indicators of creativity, tend to increase. 
Table 1

Data Obtained on SPBI and Linkographic entropies

\begin{tabular}{|c|c|c|c|c|c|c|c|c|}
\hline Participants & $\begin{array}{l}\text { SPBI } \\
\text { Score }\end{array}$ & $\begin{array}{l}\text { SPBI } \\
\text { Score } \\
\text { Mean } \\
\end{array}$ & Absolute & Relativistic & Dialectic & $\begin{array}{c}\text { Total } \\
\text { Segments }\end{array}$ & $\begin{array}{l}\text { Total } \\
\text { Links }\end{array}$ & $\begin{array}{l}\text { Link } \\
\text { Index }\end{array}$ \\
\hline Participant1 & 55 & 2,037 & 5 & 16 & 6 & 59 & 114 & 1,93 \\
\hline Participant2 & 58 & 2,148 & 4 & 15 & 8 & 42 & 85 & 2,02 \\
\hline Participant3 & 58 & 2,148 & 3 & 17 & 7 & 103 & 207 & 2,01 \\
\hline Participant4 & 59 & 2,185 & 6 & 10 & 11 & 65 & 119 & 1.83 \\
\hline Participant5 & 60 & 2,222 & 3 & 15 & 9 & 36 & 74 & 2.06 \\
\hline Participant6 & 60 & 2,222 & 3 & 15 & 9 & 100 & 206 & 2,06 \\
\hline Participant7 & 60 & 2,222 & 6 & 9 & 12 & 151 & 314 & 2,08 \\
\hline Participant8 & 60 & 2,222 & 6 & 9 & 12 & 67 & 139 & 2,07 \\
\hline Participant9 & 61 & 2,259 & 1 & 18 & 8 & 128 & 281 & 2,20 \\
\hline Participant10 & 61 & 2,259 & 5 & 10 & 12 & 149 & 324 & 2,17 \\
\hline Participant11 & 62 & 2,296 & 1 & 17 & 9 & 120 & 278 & 2,32 \\
\hline Participant12 & 62 & 2,296 & 2 & 15 & 10 & 127 & 295 & 2,32 \\
\hline Participant13 & 63 & 2,333 & 1 & 16 & 10 & 102 & 275 & 2,70 \\
\hline Participant14 & 63 & 2,333 & 3 & 12 & 12 & 84 & 206 & 2.45 \\
\hline Participant15 & 63 & 2,333 & 3 & 12 & 12 & 82 & 195 & 2,38 \\
\hline Participant16 & 63 & 2,333 & 3 & 9 & 14 & 134 & 293 & 2,19 \\
\hline Participant17 & 64 & 2,370 & - & 17 & 10 & 120 & 294 & 2.45 \\
\hline Participant18 & 66 & 2,444 & - & 15 & 12 & 119 & 305 & 2,56 \\
\hline Participant19 & 66 & 2,444 & 4 & 7 & 16 & 120 & 273 & 2.28 \\
\hline Participant20 & 68 & 2,518 & 1 & 11 & 15 & 221 & 607 & 2,75 \\
\hline Participant21 & 68 & 2,518 & 1 & 11 & 15 & 146 & 400 & 2,74 \\
\hline Participant22 & 68 & 2,518 & - & 13 & 14 & 225 & 626 & 2,78 \\
\hline Participant23 & 69 & 2,555 & 2 & 8 & 17 & 139 & 412 & 2,96 \\
\hline Participant24 & 70 & 2,592 & - & 11 & 16 & 224 & 718 & 3,21 \\
\hline Participant25 & 71 & 2,629 & 1 & 8 & 18 & 135 & 464 & 3,44 \\
\hline
\end{tabular}

Table 2 shows the correlation matrix of the SPBI scores and the Linkographic entropies. 
Table 2

Correlation matrix of SPBI and Linkographic entropies

\begin{tabular}{lrrrrrr}
\hline & Link_index & SPBI_Score & Absolute & Relativistic & Dialectic & CM6+ \\
\hline Link_index & 1 & .923 & -.681 & -.291 & .745 & .880 \\
SPBI_Score & .923 & 1 & -.602 & -.446 & .879 & .858 \\
Absolute & -.681 & -.602 & 1 & -.317 & -.198 & -.536 \\
Relativistic & -.291 & -.446 & -.317 & 1 & -.818 & -.391 \\
Dialectic & .745 & .879 & -.198 & $-.818^{* *}$ & 1 & .760 \\
CM6+ & .880 & .858 & -.536 & -.391 & .760 & 1 \\
\hline
\end{tabular}

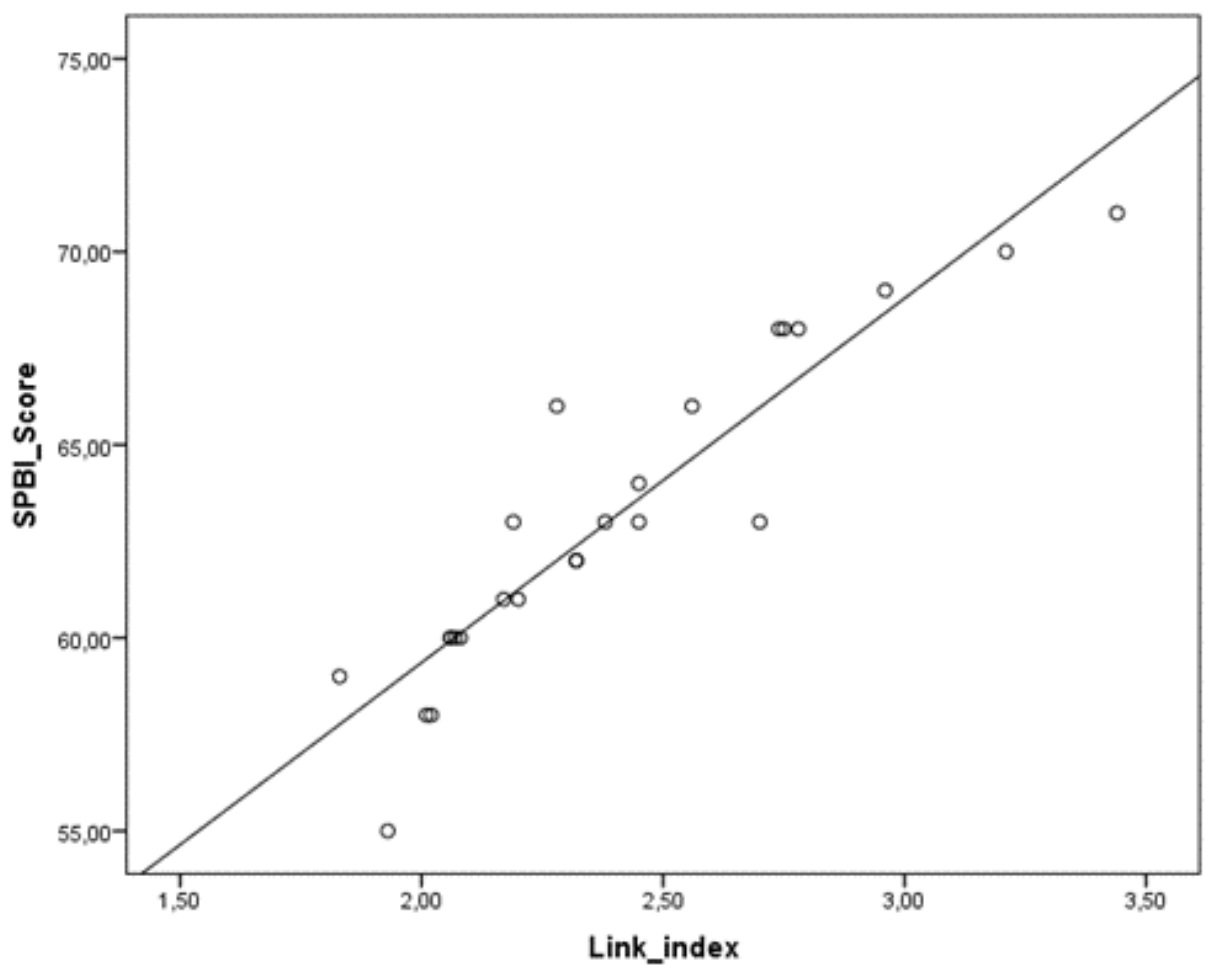

Figure 4. Correlations between Link indexes and SPBI scores.

Figure 4 presents the correlation between participants' SPBI scores and the link indexes. As shown in Figure 4, there is a positive correlation between the link indexes and SPBI scores. The coefficient of correlation; $r=.923$, was measured at the .01 level. 


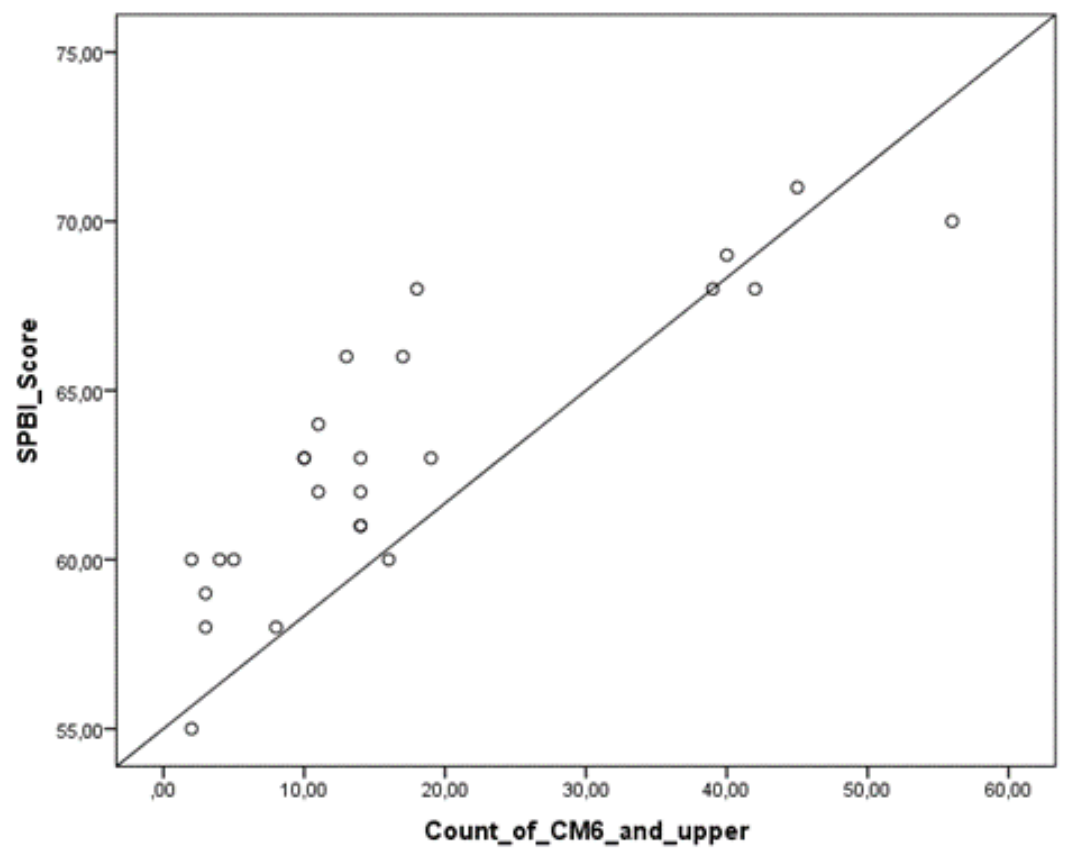

Figure 5. Correlations between SPBI Score and number of CM6+.

When the SPBI scores were correlated with the critical moves which created at least six links (CM6+), given in Figure 5, it was found that these two variables strongly correlated with each other, $r=.858$.

To further assess the relations between creativity and thinking levels, link indexes and critical moves which created at least six links were matched separately against absolute, relativistic and dialectical thinking scores.

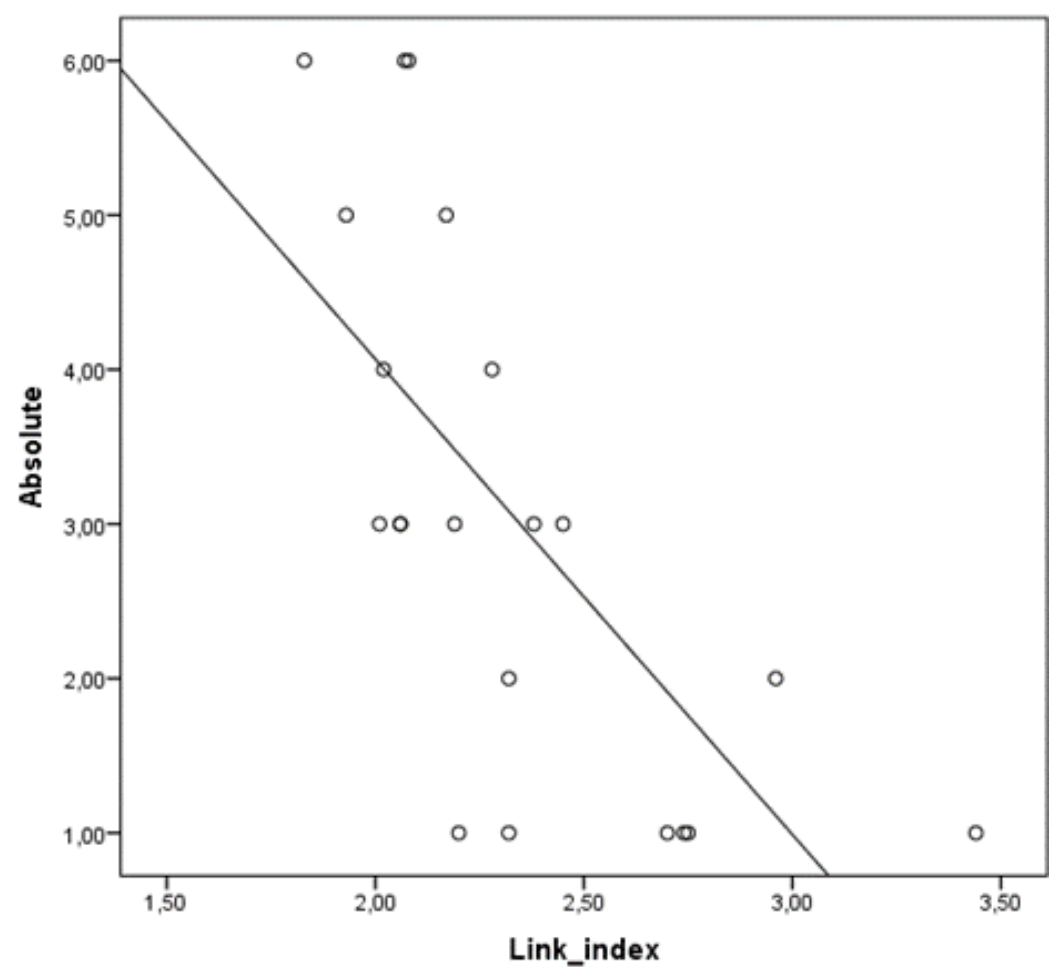

Figure 6. Correlations between Link indexes and number of Absolute statements chosen. 
As can be seen in Figure 6, there is a statistically significant negative correlation; $r=-.681(p<.01)$, between absolute thinking and link indexes.

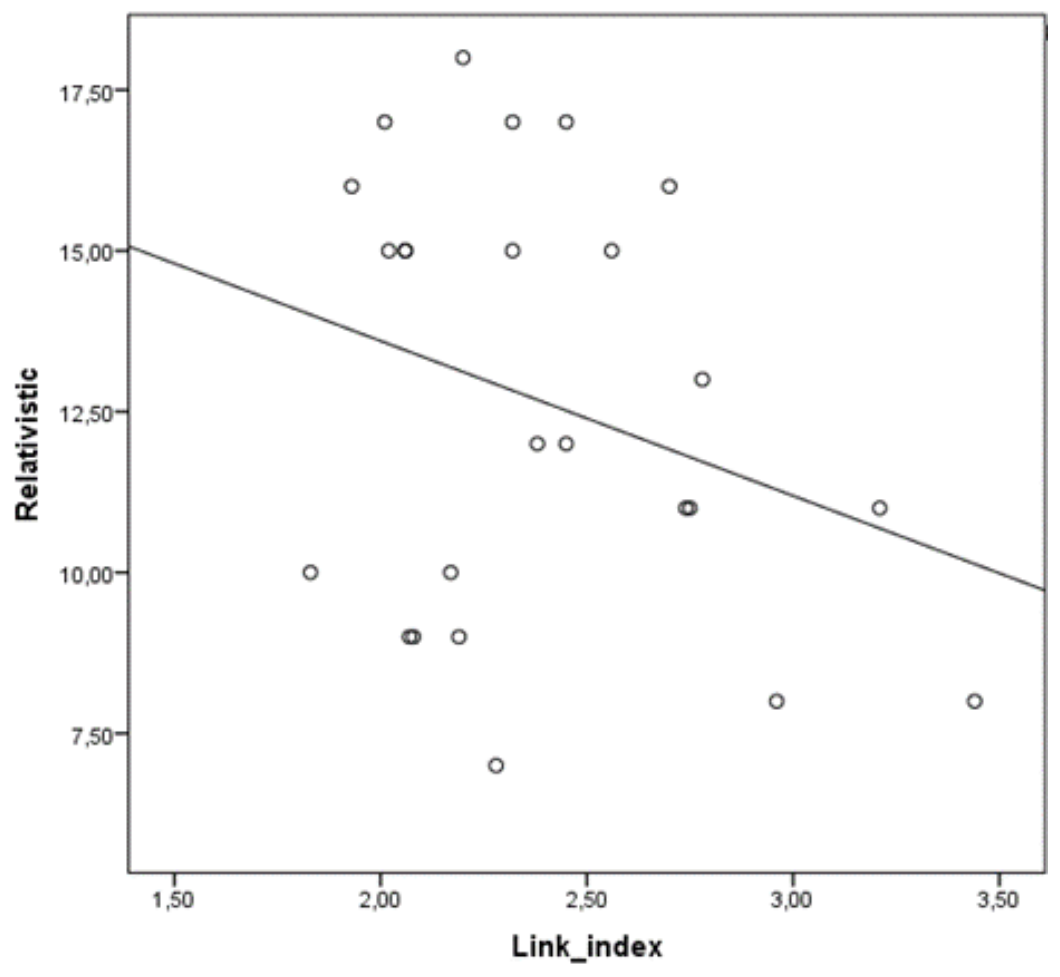

Figure 7. Correlations between Link indexes and number of chosen relativistic statements.

Results of the comparison between link indexes and relativistic thinking showed a weak negative relation; $r=-.291$ (See Figure7).

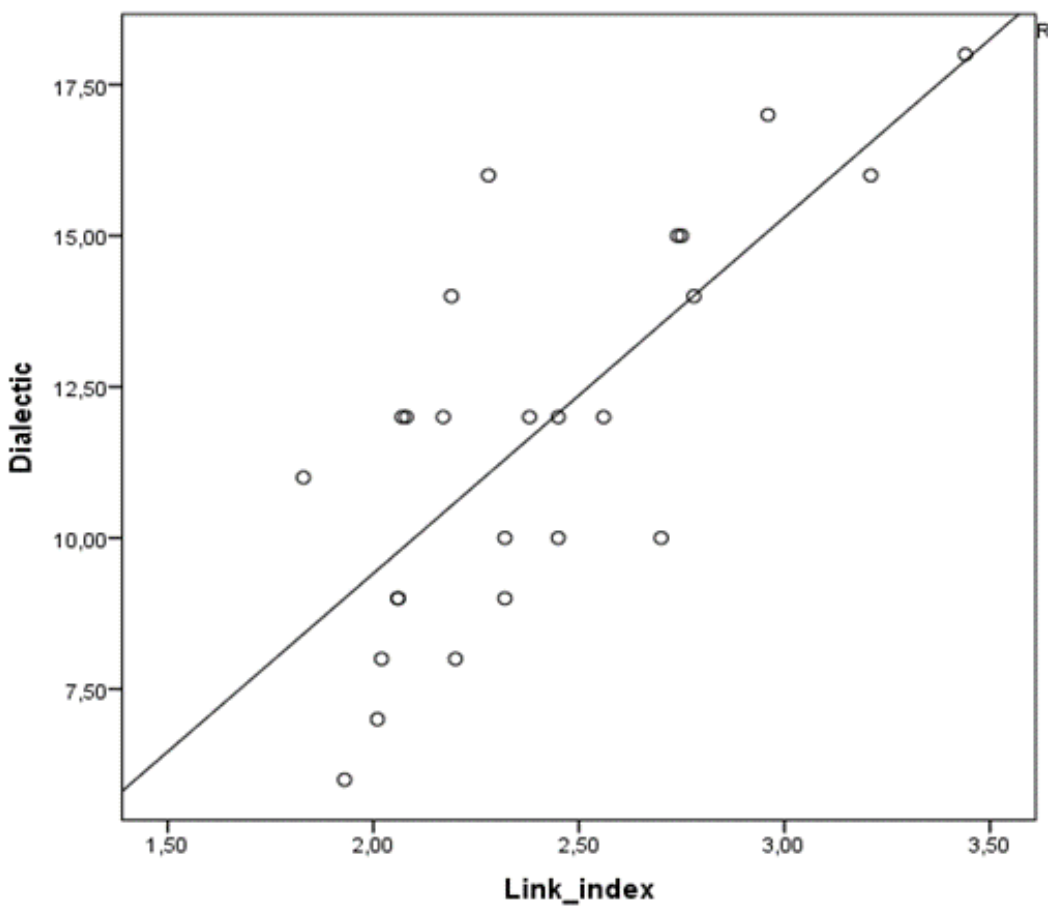

Figure 8. Correlations between Link indexes and the number of chosen dialectic statements. 
As expected, the correlations between link indexes and dialectical thinking showed a significant positive relation; $r=.745$ (See Figure 8).

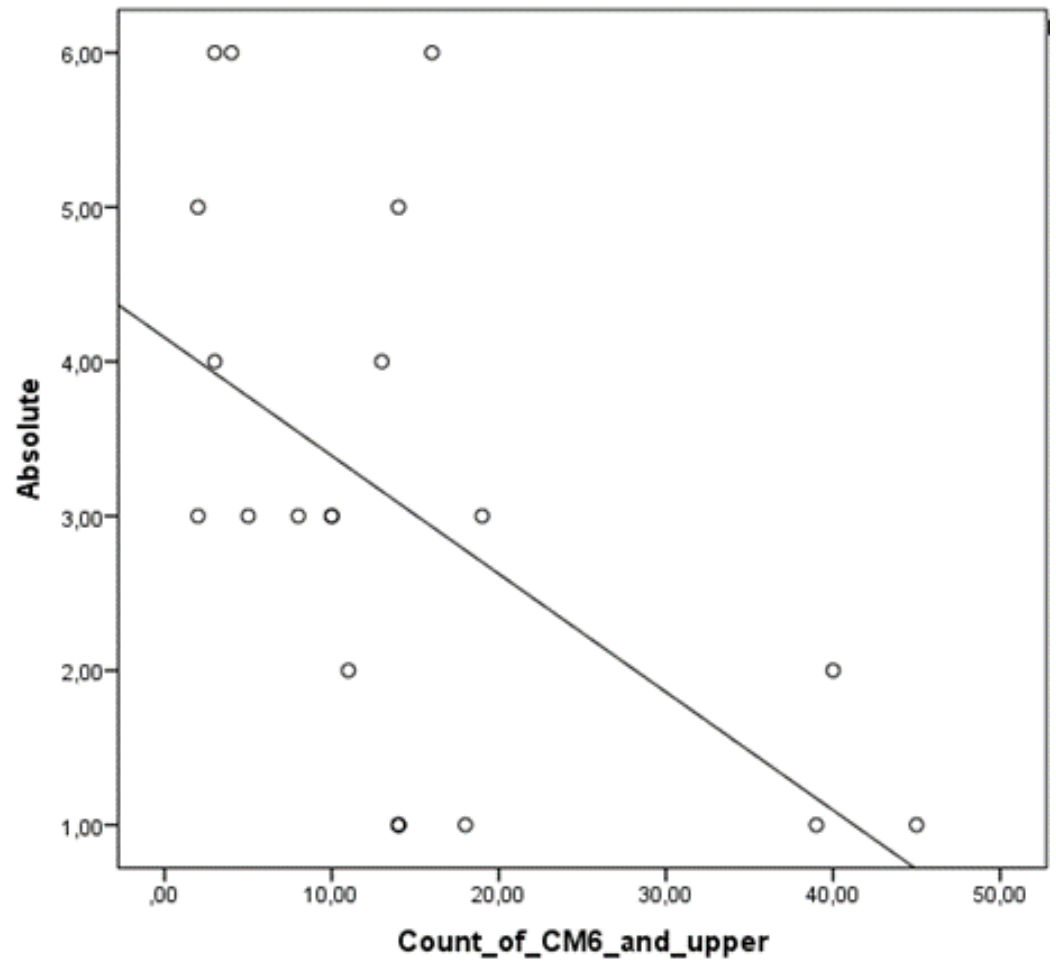

Figure 9. Correlations between $\mathrm{CM} 6+$ and the number of chosen absolute statements.

Results of the comparison between $\mathrm{CM} 6+$ and absolute thinking showed a significant negative relationship; $r=-.536$ (See Figure 9).

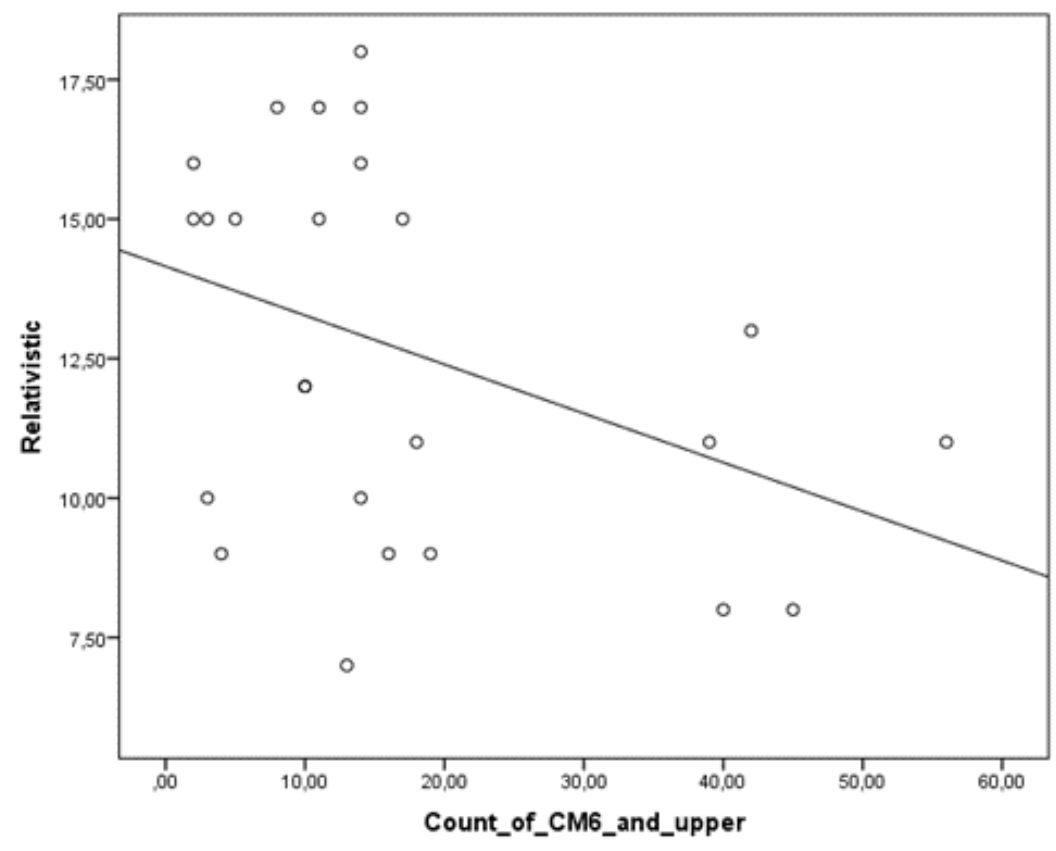

Figure 10. Correlations between $\mathrm{CM} 6+$ and the number of chosen relativistic statements. 
When the $\mathrm{CM} 6+$ scores were correlated with the number of chosen relativistic statements, given in Figure 10, it was found that these two variables weakly correlated with each other, $r=-.391$.

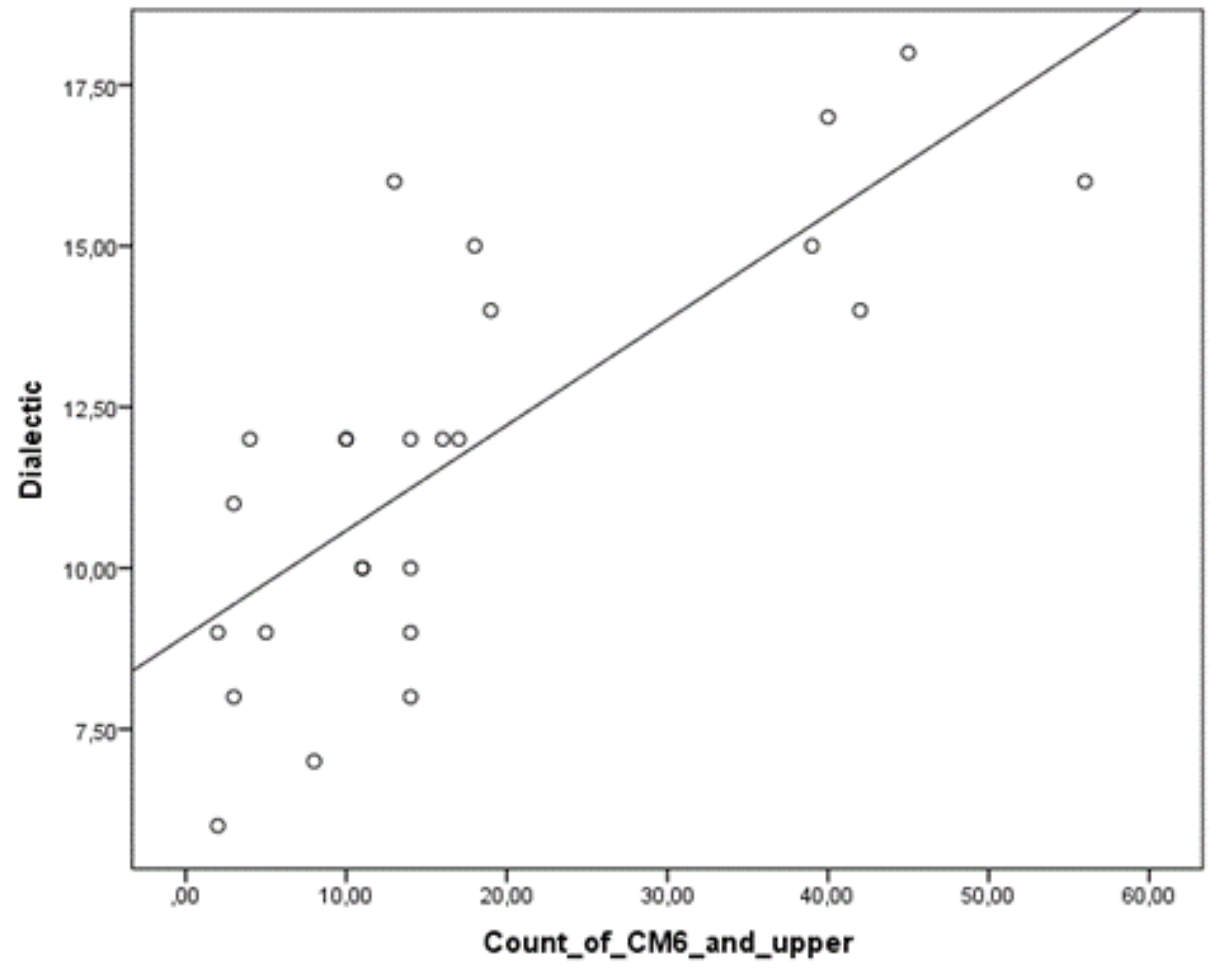

Figure 11. Correlations between $\mathrm{CM} 6+$ and the number of chosen dialectical statements.

Figure 11 presents the correlation between CM6+ scores and dialectical thinking. As expected, there is a positive correlation between scores. The coefficient of correlation; $r=.760$, was measured at the .01 level.

\section{DISCUSSION AND CONCLUSION}

The hypothesis examined in this study was that participants at post-formal thinking levels would obtain good link index and critical move scores, that is, that they would create more links in order to find creative solutions to ill-defined design problems. To investigate this, the participants' SPBI scores were compared with the total number of links created during the design sessions. The results showed that people with high SPBI scores tend to have high link index values, which seems to indicate post-formal thinkers tend to create more ideas and links, and thus tend to be creative. As high SPBI scorers are better at perceiving actual or potential relations between previously unrelated matrices of thought, the high scorers might have more ideas to link with each other, even if the previous unrelated ideas are contradictory. Similarly, it has been shown that having a higher SPBI score has a significant relationship with having a high number of critical moves. This might be because post-formal thinkers are aware of their enquiry process, they tend to build denser linking structures by linking as many design moves to each other, in order to verify their thinking process. 
As expected, the research has shown that formal thinking has a negative correlation with the productivity of linking. Results showed that Absolute thinking has a strong negative correlation with both link index and critical moves. These results echo previous work in which formal thinking was negatively related to creativity (Blouin \& McKelvie, 2012; Yang, Wan \& Chiou, 2010; Wu \& Chiou, 2008). As formal thinkers tend to work in closed systems, creating more links might actually be challenging. Hence, they are unable to break open common beliefs and create more creative, unconventional solutions.

In this study, a weak positive correlation was expected between link indexes and relativistic thinking. However, the results show a weak negative correlation with creativity. Similarly, relativistic thinking showed a weak correlation with critical moves. Although relativistic thinking is a thought process within the post-formal thought stage, and as such should be positively correlated with creativity, it is also a competitor of dialectical thinking, which is even more strongly correlated with creativity. Indeed research results indicate that relativistic thinking might have a regressive effect on creativity by inhibiting dialectical thinking. As can be seen in Table 2, relativistic thinking has a strong negative correlation with dialectical thinking; $r=-.818$. In addition to this, relativistic thinking showed a significant negative relation with SPBI scores; $r=-.681$. As the Relativistic thinkers tend to create new ideas by linking knowledge units within a dependently changing thought process and cannot work with multiple perspectives at once, they might suffer from the inability to structure their thought processes well. As an example, Participant 9, with the highest number of chosen relativistic statements and Participant 19, with the lowest number of chosen relativistic statements had linkographs that are presented below; as seen in the figures, Participant 9 has a dispersed and poorly structured thought process, whereas Participant 19's linkograph is clustered and better structured.

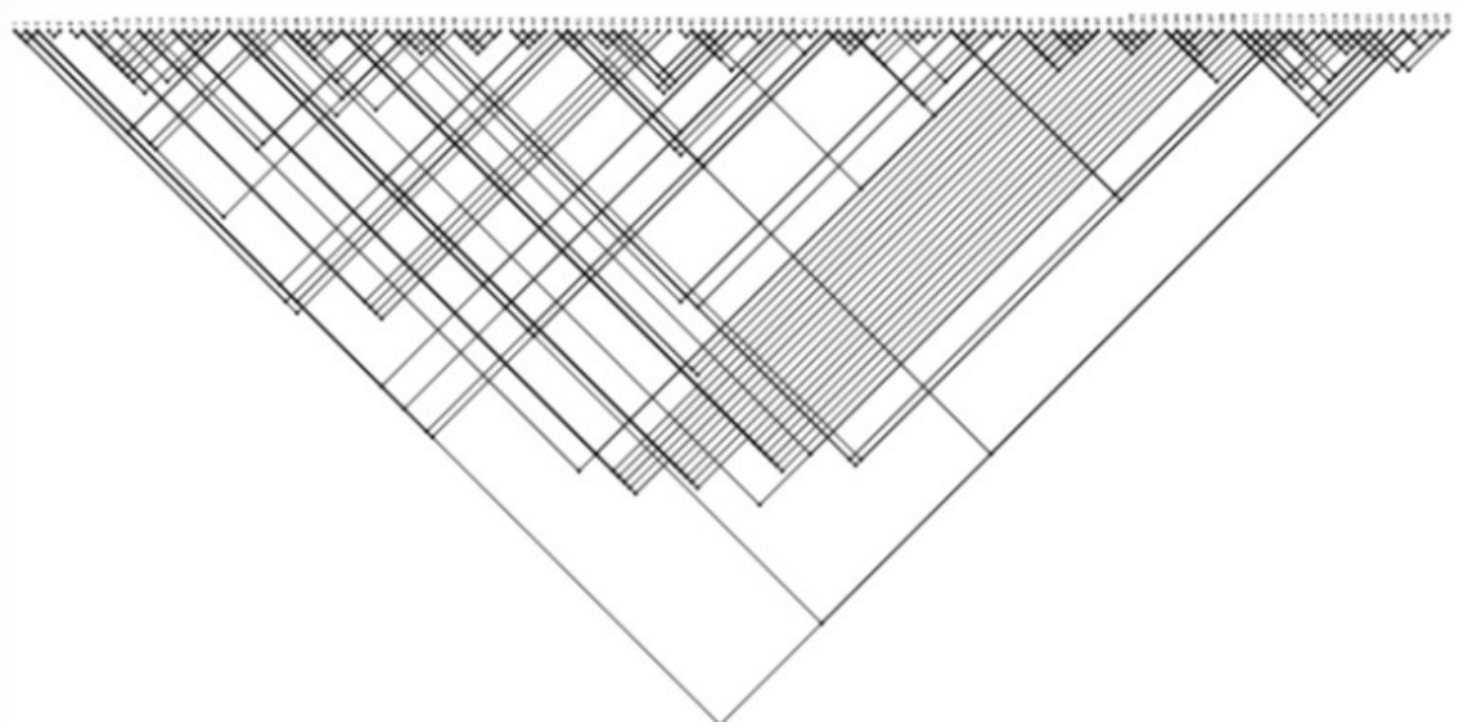

Figure 12. Linkograph of participant 9. 


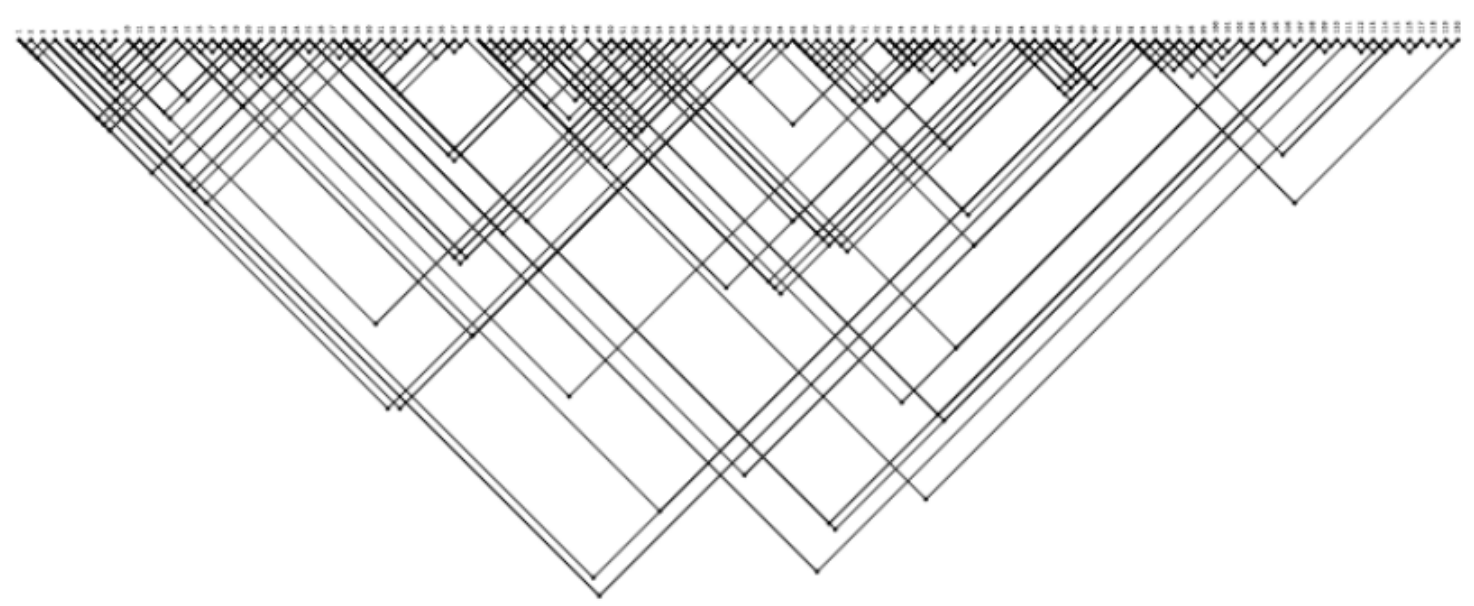

Figure 13. Linkograph of participant 19.

As was expected, the correlations between link indexes and dialectical thinking showed a significant positive relationship. Similarly, as was also expected, the results showed that there is a strong positive correlation with dialectical thinking and critical moves. This could indicate that people who have the ability to link knowledge across multiple perspectives at a given point in time and to comprehend contradictions tend to be more creative. Also, as they are able to connect more independent knowledge chunks and able to work with multiple perspectives at one time, they are better at structuring their thought processes and creating possible novel and applicable potential solutions.

From these results, it is possible to arrive at the conclusion that dialectical thinking is more useful for finding increasingly novel solutions to design problems and being creative, whereas relativistic thinking and formal thinking are negatively associated with these abilities. The results obtained from this research also show that the SPBI scores may be used as determinants of individuals' creativity as they showed strong relationships with idea generation productivity and linking activity.

Future studies may investigate the roles of relativistic and dialectical thinking independently from formal thinking, in order to further assess why relativistic thinking negatively correlated with linking productivity and creativity. In order to achieve this, researchers may need a different thinking style measurement, other than the SPBI, just focusing on relativistic and dialectical thinking.

\section{REFERENCES}

Akin, Ö. \& Akin, C. (1996). Frames of reference in architectural design: Analysing the hyper-acclamation (Aha!). Design Studies, 17(4), 341-361.

Basseches, M. (1984). Dialectical thinking and adult development. Norwood, NJ: Ablex. 
Basseches, M. (1989). Dialectical thinking as an organized whole: Comments on Irwin and Kramer. In M. L. Commons, J. D. Sinnott, F. A. Richards, \& C. Armon (Eds.), Adult development: Comparisons and applications of developmental models (pp. 161-178). New York: Praeger.

Blouin, P. S. \& McKelvie, S. J. (2012). Postformal thinking as a predictor of creativity and of the identification and appreciation of irony and metaphor. North American Journal of Psychology, 14(1), 39-50.

Boden, M. A. (1994). What is creativity? In M. A. Boden (Ed.), Dimensions of creativity (pp. 75-117). Cambridge: MIT Press.

Commons, M. L, Bresette L. M., \& Ross S. N. (2008). The connection between postformal thought and major scientific innovations, world futures. The Journal of Global Education, 64(5-7), 503-512.

Commons, M. L. \& Richards F. A. (2003). Four Postformal Stages. In J. Demick and C. Andreoletti (Eds.), Handbook of Adult Development. The Springer Series in Adult Development and Aging, Book Section (pp. 199-219). US: Springer.

Cross, N. (1997). Descriptive models of creative design: Application to an example. Design Studies 18(4), 427-455.

Cross, N. (2008). Engineering design methods: Strategies for product design (4th ed.). Chichester: John Wiley \& Sons.

Gabora, L. (2002). Cognitive mechanisms underlying the creative process. In Proceedings of the 4th conference on Creativity \& cognition (pp. 126-133). ACM.

Goldschmidt, G. (2014). Linkography: Unfolding the design process. MIT Press.

Goldschmidt, G. \& Tatsa D. (2005). How good are good ideas? Correlates of design creativity. Design Studies, 26 (6), 593-611.

Gürsoy, B. (2010). The cognitive aspects of model-making in architectural design. (Unpublished MSc thesis). Turkey: Middle East Technical Unıversity.

Kramer, D. A. (1983). Post-formal operations? A need for further conceptualization. Human Development, 26, 91-105.

Kramer, D. A. (1989). Development of an awareness of contradiction across the life span and the question of postformal operations. In M. L. Commons, J. D. Sinnott, F. A. Richards, and C. Armon (Eds.), Adult development Comparisons and applications of developmental models. (pp. 133-159). New York: Praeger.

Kramer, D. A. (1992). The Social Paradigm Belief Inventory. 3 Choice- Forced Choice Version. [PDF document]. Retrieved from http://www.deirdrekramer.com/ Dr._Deirdre_Kramer/The_Social_Paradigm_Belief_Inventory_files/Social\% 20Paradigm\%20Belief\%20Inventory\%20--\%20SPBI\%20--\%20Forced\%20Version.pdf 
Kramer, D. A., Kahlbaugh, P. E., \& Goldston, R. B. (1992). A measure of paradigm beliefs about the social world. Journal of Gerontology: Psychological Sciences 47(3), 180-189.

Koestler, A. (1960). The Act of Creation. New York: Macmilla.

Labouvie-Vief, G. (1980). Beyond formal operations: Uses and limits of pure logic in lifespan development. Human Development, 23(3), 141-161.

Laske, O. (1999). An integrated model of developmental coaching. Consulting Psychology Journal, 51(3), 139-159.

Marchand, H. (2002). Some reflections on postformal stage. Behavioral Development Bulletin, 11(1), 39-46.

McBride, L. R. (1998). A comparison of postformal operations in diverse adult populations: Contrasting African-Americans and standard average European-Americans (working class). (Unpublished doctoral dissertation) Texas Tech University, US.

Mumford, M. D., Mobley, M. I., Uhlman, C. E., Reiter-Palmon, R., \& Doares, L. M. (1991). Process analytic models of creative capacities. Creativity Research Journal, 4, 91-122.

Mwamwenda, T. (1993). Formal operations and academic achievement. The journal of psychology, 127(1), 99-103.

Perry, W. G. (1981). Cognitive and ethical growth: The making of meaning. In A. Chickering (Ed.), The modern American college (pp. 76-116). San Francisco: Jossey-Bass.

Piaget, J. (1958). Growth of logical thinking from childhood to adolescence. New York: Basic Books.

Pourmohamadi, M., \& Gero, J. S. (2011). LINKOgrapher: an analysis tool to study design protocols based on FBS coding scheme. In DS 68-2: Proceedings of the 18th International Conference on Engineering Design (ICED 11), Impacting Society through Engineering Design, Vol. 2: Design Theory and Research Methodology, Lyngby/ Copenhagen, Denmark, 15.-19.08. 2011.

Runco, M. A. (1994). Creativity and its discontents. In M. P. Shaw, and, M. A. Runco (Eds.). Creativity and affect (pp. 102-123). Westport, CT, US: Ablex Publishing.

Runco, M. A. (2004). Creativity. Annual Review of Psychology, 55, 657-687.

Santanen, E. L., Briggs, R. O., \& de Devreede, G. J. (2002). Toward an understanding of creative solution generation. In System Sciences, 2002. HICSS. Proceedings of the 35th Annual Hawaii International Conference on (pp. 2899-2908). IEEE.

Schön, D. A. (1983). The Reflective Practitioner: How Professionals Think in Action. New York: Basic Books.

Sinnott, J. D. (1989). Everyday problem solving: Theory and application. New York: Praeger. 
Someren, M., Barnard, Y., \& Sandberg, J. (1994). The Think Aloud Method: A Practical Guide to Modelling Cognitive Processes. London: Academic Press.

Tatsa, D. (2005). Ideas in students' projects in the architectural studio: their sources, development, roles and assortment. (Unpublished MSc thesis), Technion, Israel.

Torrance, E. P. (1988). The nature of creativity as manifest in its testing. In R. J. Sternberg (Ed.), The nature of creativity (pp. 43-75). Cambridge: University Press.

Torrance, E. P., \& Presbury, J. (1984). The criteria of success used in 242 recent experimental studies of creativity. Creative Child and Adult Quarterly, 9, 238-243.

Valanides, N. (1998), Formal operational performance and achievement of lower secondary school students. Studier in Educational Evaluation, 24(1), 1-23.

van der Lugt, R. (2003). Relating the quality of the idea generation process to the quality of the resulting design ideas. In DS 31: Proceedings of ICED 03, the 14th International Conference on Engineering Design, Stockholm.

Vanier, D. Searight, H.R. (2013). The Social Paradigm Belief Inventory (SPBI): A factor analytic study. Modern Behavioral Science, 1(1), 49-56.

Wallas, G. (1928). The art of thought. New York: Harcourt-Brace.

Ward, T.B., Smith, S.M. \& Vaid, J. (1997). Conceptual structures and processes in creative thought. In T.B. Ward, S.M. Smith, and J. Vaid, (Eds.), Creative thought: An investigation of conceptual structures and processes. (pp. 1-27) American Psychological Association.

Wu, P-L. \& Chiou W-B. (2008). Postformal thinking and creativity among late adolescents: A post-Piagetian approach. Adolescence, 43(170), 237-251.

Yang, C-C., Wan, C-S. \& Chiou, W-B. (2010). Dialectical thinking and creativity among young adults: A postformal operations perspective. Psychological Reports, 106(1), 1-14.

Corresponding author at: Nazife Asli Kaya, Izmir Institute of Technology, Faculty of Architecture, Department of Industrial Design Urla/ Izmir, Turkey, Postal code: 35430.

E-mail: n.aslikaya@gmail.com

(C) Copyright by Faculty of Pedagogy and Psychology, University of Bialystok,

20 Swierkowa St., 15-328 Bialystok, Poland tel. +48857457283 